\title{
Virtual Cell Zooming and Sleep Mode for 3GPP-LTE Green Cellular Networks
}

\author{
Bruno Saldanha Carminati, Marcelo Faleiros Costa, and André Noll Barreto
}

\begin{abstract}
This paper presents a study on green cellular networks for 3GPP-LTE networks, comparing the performance of the sleep mode and cell zooming algorithms not only in terms of their energy efficiency, but also verifying whether energy savings do not cause coverage or throughput losses in the network. In order to perform this study, we have developed a radio network simulator, which accounts for the traffic variation of a cellular network over the day and over different regions of the grid. Because of performance and implementation limitations of the cell zooming algorithm found in the literature, we propose a modification, called virtual cell zooming.
\end{abstract}

Keywords-Cell Zooming, Energy Efficiency, Green Cellular Networks, Power Management.

\section{INT RODUCTION}

$\mathrm{I}_{\mathrm{s}}^{\mathrm{N}}$ recent years, traffic demand in cellular networks has significantly grown due both to popularization of several types of services and to the technology evolution, which led to higher capacity and lower costs. Even though battery consumption of handsets has always been a major concern, so far throughout the expansion of mobile networks, there has not been much effort in assessing and reducing energy consumption on the network side of cellular networks. However, recently, an increasing worldwide concern about potential damages to the environment, such as carbon dioxide emissions and depletion of non-renewable energy resources, as well as rising operational costs and lower profit margins, impelled many researchers to study different ways on how to minimize energy consumption of cellu lar systems.

It is estimated that $2 \%$ of world's annual emissions of $\mathrm{CO}_{2}$ are caused by the information and communication infrastructure, and the tenth part of that relates to cellular networks [1]. We should however bear in mind that due to the exponential growth in mobile networks, energy efficiency is a key aspect to make sure that emissions remain low in a foreseeable future.

Besides that, not only the environmental aspects are relevant, but also the economical ones, since operational expenditures of radio base stations (RBSs) connected to the electrical grid may reach US $\$ 3000$ per year, while off-grid RBSs, located in remote areas and running on diesel power generators, may cost ten times more [2].

For all these reasons, cellular network operators explore new ways to improve energy efficiency, and the interest of

Bruno Saldanha Carminati, Marcelo Faleiros Costa, and André Noll Barreto are with the Department of Electrical Engineering, University of Brasília, Distrito Federal, Brazil (e-mails: \{brunoscarminati, marcelofaleiros\} @aluno.unb.br, andrebarreto@ene.unb.br). This work was partially supported by the Dean of Undergraduate Studies of the University of Brasília. researchers is focused in a new research area called "Green Cellular Networks" [2]. Hence, the goal of this paper is to analyze and suggest solutions to render cellular communication networks less harmful to the environment.

Two key elements are identified when we consider energy consumption on cellular networks: the great number of radio base stations deployed and traffic fluctuations during the day or the week. RBSs are responsible for almost $60 \%$ of the overall energy consumption of cellular systems [2]. Therefore, effort must be directed to reducing RBSs' consumption.

In traditional cellular networks, cell size and capacity are specified during network design, according to peak traffic estimates for a specific area. However, time and spatial traffic fluctuations may render the project inefficient, demanding a more dynamic network, capable of adjusting itself constantly. There is an enormous potential for energy savings on cellular systems if during low demand periods some RBSs are switched off. Unfortunately, currently this potential is usually not seized. Moreover, energy efficiency should not come at the expense of reduced system performance and user experience [1]. As next cellular generations move towards deployment of smaller cells, such as micro-, pico-, and femto-cells, traffic fluctuations may be even higher [3].

With this in mind, the concept of cell zooming [3] was created: a power management technique in which base stations adjust cell size according to traffic situation. The main goal is to balance traffic demand, and at the same time enhance system performance or reduce energy consumption. This technique seems to be a very promising solution, although it is not being commercially implemented yet. Some studies show that cell zoo ming can save up to $40 \%$ energy [2].

In this paper, we focus the study of energy efficiency through power management of fourth generation networks, since the 3GPP-LTE standard is the latest and most recently released. In order to investigate this, we implemented a network simulator that models a dynamic traffic behavior and collects energy consumption and performance data to demonstrate energy efficiency reached with and without cell zoo ming. In addition, our proposal is to separate the technique into two: sleep mode and virtual cell zooming. The sleep mode is a specific type of cell zooming, in which cells can zoom in to zero, meaning they can be switched off and temporarily consume no energy. The virtual cell zooming is a modification of the conventional cell zooming, in which cells change sizes with no physical adjustments. While conventional cell zoo ming aims at energy efficiency only, virtual cell zoo ming is focused in system performance and better resource distribution. In our proposal, energy efficiency is obtained by sleep mode, whereas virtual cell zooming helps improving or maintaining the performance of the cellular network. 
In Section II we discuss the concept, is sues and paradigms of green cellular networks, as well as briefly explain the cell zooming technique. Section III expands the concepts of the proposed techniques, where we discuss their usage, aspects, and expected results. System modeling and formulation are presented in Section IV. Results are discussed in Section V and conclusions are drawn in Section VI.

\section{ASPECT S OF GREEN CELLULAR NETWORKS AND A FIRST BRIEFING ON CELL ZOOMING}

To typify cellular networks as green or not, we would naturally evaluate carbon footprint or $\mathrm{CO}_{2}$ emissions. The share of emissions for cellular networks is relatively low, making it not an appropriate metric in this case [2]. On the other hand, the notion of green can be evaluated by economical and energy efficiency aspects. A set of practical metrics should provide necessary information about system operation, energy savings, and performance [2]. We define four metrics: number of mobile users per cell, users' downlink throughput, outage probability and average consumed power per base station.

The number of mobile users per cell is a control metric, which acts as a basis parameter to evaluate all others, defining when traffic is more or less intense.

The performance is evaluated by users' throughput and outage probability, whereas the latter refers to users whose received signals are below sensitivity level and cannot connect to any base station. These metrics are needed to assure that user experience is not degraded because of energy savings.

Finally, energy efficiency is measured by average consumed power per base station.

As it is known, traffic fluctuations can be significant during a day or a week. At daytime, traffic load is heavier in commercial areas when compared with residential sites; at night, the situation is opposite. Hence, there are always RBSs under low traffic, while others experience high demand [3].

This is how the concept of cell zooming plays a major role. A congested cell should zoom in, i.e., decrease in size, while its neighboring cells could zoom out and absorb mobile users the former could not do. In some cases, the congested cell can even be switched off, the so-called sleep mode, drastically reducing power consumption.

Changes in the size of a cell are mainly done by physical adjustments. Cells can zoom in or out by reducing or increasing the RBS transmit power, or by adjusting antenna height and tilt. Also, when a cell zooms in to zero and enters into sleep mode, the air-conditioner, the power amplifier, and other energy consuming devices can be switched off.

\section{CELL ZOOMING SERVER}

An element needed to implement the technique is the cell zoo ming server, which may be either centralized or distributed over all base stations [3]. The server senses network state information, and performs necessary decisions about power management. A self-organizing server is required since the system itself should be able to make adjustments without operational staff intervention. Nonetheless, automatic cell zooming may cause inter-cell interference and coverage holes [3], requiring further studies to overcome every challenge.

In the following, we present the proposed techniques, and how the server executes its actions.

\section{A. Sleep Mode}

Usually, sleep mode algorithms choose automatically which cells will be switched off, and the criterion is to choose cells with the smallest amounts of users. However, the existence of hotspots in the grid concentrates users in some areas, while other areas may experience low traffic load. Hence, it is probable that an arbitrary choice results in uncovered regions.

To avoid such problem, one must observe initial positioning of cells throughout the grid. We propose switching off 3 out of each 4 cells. Also, as in this paper we consider RBSs deployed with directional antennas, $(3,4)$-off scheme is the only feasible one [4], since cell direction does not change. Considering transmit power increase and tilt adjustment, very few areas remain uncovered, as we can see in Fig. 1.

It is clear that the sleep mode exploits time asymmetry of a cellular network and must be activated when the number of users per cell is smaller than a certain value, which happens during light traffic load.

\section{B. Virtual Cell Zooming}

Besides time asymmetry, cellular networks are also spatially asymmetric. Because of this, traffic is unevenly distributed, and users connected to heavier loaded cells experience service with lower quality than those in emptier cells.

When the conventional power management of cell zooming acts, in which cells zoom in and out through physical adjustments, overloaded cells are forced to reduce their transmit power to absorb a smaller quantity of mobile users, whereas other cells must compensate by increasing theirs. Anyway, this compensation may not contribute to energy efficiency and in worst case cause coverage holes.

We observe that cell zooming can operate in a different and much more efficient way in terms of system performance. When a mobile user initiates a call, the connection is usually made with the base station from which received power level is higher at the mobile device. Despite that, a considerable number of neighboring base stations may also be able to serve that user. At this moment, the cell zooming server may act to reconnect the user to a lightly loaded cell instead, if received power is superior to device sensitivity.

The action of the server consists in sweeping all neighboring

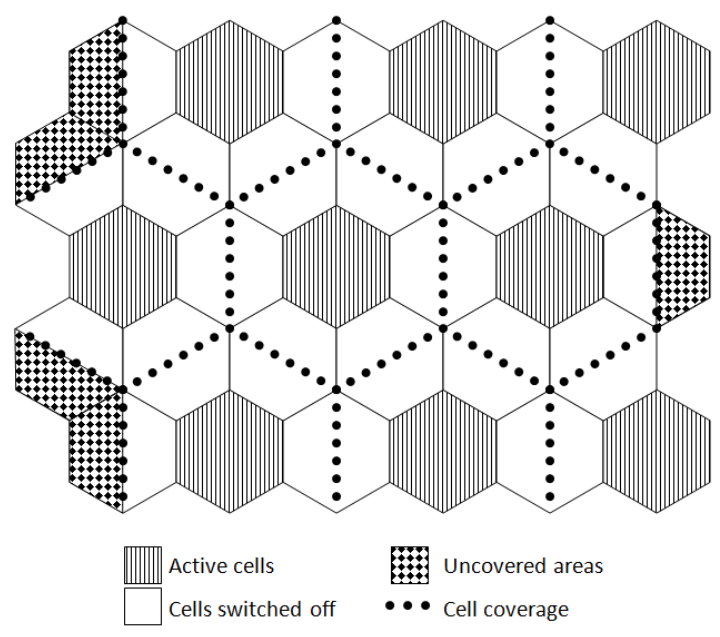

Fig. 1. Rectangular grid of hexagonal cells and its configuration in the execution of the sleep mode; 3 out of 4 cells are inactive. 
cells in the grid and checking the number of users connected to them. If these cells serve less mobile users than the best server and their transmit power still is sufficient to provide connection from a farther distance, the user initiating the call will establish connection with the lightest loaded neighboring cell. If none of the cells fulfill the requirements of sufficient transmit power and less connected users, the user maintains connection with the best server.

In this approach, overloaded cells reduce their number of users, shedding particularly those users on their borders, which are taken up by neighboring lightly loaded cells. These cells grow in size requiring no physical changes at the network, hence the name of virtual cell zooming. These dynamic adjustments result in a much fairer resource distribution.

\section{SYSTEM MODELING}

An event-driven simulator was written in $\mathrm{C}++$ to investigate the performance of the diffe rent algorithms. The source code is publicly available from the address https://code.google.com/ p/green-cell-sim. Models and assumptions in the developed simu lator are summarized below.

\section{A. Models and Assumptions}

1) The grid consists of hexagonal tri-sector cells with 250 meter radius deployed in a rectangular arrangement, as shown in Fig. 1. To account to the fact that in real life grids are not regular, RBSs are positioned arbitrarily inside a small squared region of approximately $150 \mathrm{~m}^{2}$ in the center of each tri-s ector cell.

2) The path loss $P L_{\mathrm{dB}}$ is computed by Okumura-Hata Extended Cost-231 model for frequencies between 1,500 and 2,000 MHz and large cities environment [5].

3) We compute an additional loss due to shadowing, which is given by a shadowing factor $S F_{\mathrm{dB}}$. The shadowing factor has a log-normal distribution and different standard deviations for different scenarios. Closely positioned mobile users typically have similar or correlated SFs. Shadowing factor correlation can be modeled by interpolation as described in [6].

4) Antenna gain is computed as described in [7]. This model can be used in hexagonal deployed grids to represent authentic conditions in system simulations.

Horizontal antenna gain $G_{\mathrm{h}}$ is parameterized with a maximum gain $G_{\max }$, horizontal half-power beamwidth $H P B W_{\mathrm{h}}$, and front-to-back ratio $F B R$, and is given by

$$
G_{\mathrm{h}}(\varphi)=-\min \left(12\left(\frac{\varphi}{H P B W_{\mathrm{h}}}\right)^{2}, F B R\right)+G_{\max },
$$

where $\varphi,-180^{\circ} \leq \varphi \leq 180^{\circ}$, is the horizontal angle relative to the main beam pointing direction.

In the same way, vertical gain $G_{\mathrm{v}}$ considers the vertical halfpower beamwidth $H P B W_{\mathrm{v}}$, the side-lobe level $S L L$ and the downtilt angle $\theta_{\mathrm{tilt}}$, and is given by

$$
G_{\mathrm{v}}(\theta)=\max \left(-12\left(\frac{\theta-\theta_{\text {tilt }}}{H P B W_{v}}\right)^{2}, S L L\right),
$$

where $\theta,-90^{\circ} \leq \theta \leq 90^{\circ}$, is the vertical angle relative to the horizontal plane.

Finally, antenna gain is given by sum of both components, as in

$$
G_{\mathrm{TX}, \mathrm{dB}}(\varphi, \theta)=G_{\mathrm{h}}(\varphi)+G_{\mathrm{v}}(\theta) .
$$

5) Received power level $P_{\mathrm{RX}, \mathrm{dBm}}$ at user's site is computed as

$$
P_{\mathrm{RX}, \mathrm{dBm}}=P_{\mathrm{TX}, \mathrm{dBm}}+G_{\mathrm{TX}, \mathrm{dB}}-P L_{\mathrm{dB}}-S F_{\mathrm{dB}},
$$

where base stations transmit with power $P_{\mathrm{TX}, \mathrm{dBm}}$. We do not consider antenna gain in the mobile station reception. Mobile users will preferably connect to the base station that delivers the highest power level.

6) Supposing that an user is connected to the $i$-th cell, the downlink signal-to-interference-and-noise ratio $(S N I R)$ is

$$
S N I R=\frac{P_{\mathrm{RX}, \mathrm{i}}}{P_{\mathrm{N}}+\sum_{\mathrm{j} \neq \mathrm{i}} P_{\mathrm{RX}, \mathrm{j}}},
$$

where $P_{\mathrm{RX}, \mathrm{j}}$ is the power received from cell $j$. The interference is the sum of every received signal from all cells except the one the user is connected to, and $P_{\mathrm{N}}$ is the noise power.

7) We consider that OFDM resource blocks are equally distributed to every user in a cell, and that no channel coding is used.

For a calculated SNIR level, we can find the optimum modulation scheme among QPSK, 16-QAM and 64-QAM that achieves a given BER. The $E_{\mathrm{b}} / N_{0}$ ratio can be obtained as

$$
\left(\frac{E_{\mathrm{b}}}{N_{0}}\right)_{\mathrm{dB}} \approx S N I R_{\mathrm{dB}}-10 \log \left(N \log _{2} M\right),
$$

where $N$ is the number of occupied subcarriers, and $M$ indicates the constellation size of the modulation scheme.

As modulation schemes are chosen, we determine users' downlink throughput knowing how many resource elements inside a frame is allocated for each user. It is assumed that the downlink frame is $100 \%$ loaded. We do not investigate the uplink. A $10 \mathrm{MHz}$ LTE frame was considered with single antennas, pilot resource elements were not considered for the throughput calculation, and no further signaling was taken into account.

In our simulations, the maximum downlink throughput permitted per user is limited to $5 \mathrm{Mb} / \mathrm{s}$.

8) RBSs consume something between 800 e 1,500 W, split into power supply, power amplifier, signal processing and air conditioning [2]. This assumption is described in Fig. 2. Note that signal processing consumption is proportional to the number of users connected to a base station.

9) Users arrival process is modeled as a Poison process with mean arrival rate $u p s(t)$ changing with time $t$ in a sinusoidal profile, similar to the one described in [1]:

$$
u p s(t)=u p s_{\max }\left(1-\cos \left(\frac{2 \pi t}{T}+\tau\right)\right)^{\alpha},
$$

where $u p s_{\max }$ is the maximum number of users arriving per second, $T$ is the time period (e.g. a day), $\tau$ is a shift to adjust peak time, and $\alpha$ is a fit adjustment on the curve.

10) Users move throughout the grid. There are three

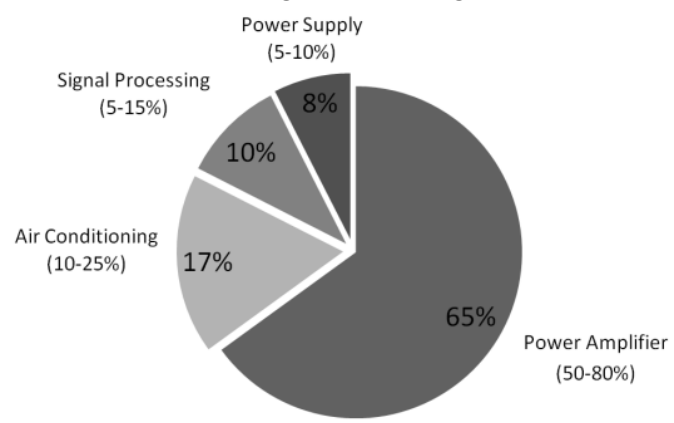

Fig. 2. Power consumption distribution in radio base stations. 
patterns of movement: Brownian (between 0.1 and $1.2 \mathrm{~m} / \mathrm{s}$ ), pedestrian (between 1.2 and $6.0 \mathrm{~m} / \mathrm{s}$ ), and vehicular (between 6.0 and $16 \mathrm{~m} / \mathrm{s}$ ). Brownian movement is characterized by complete uncorrelated directions users as sume each second; pedestrians may or may not orthogonally change their direction each second; vehicles do not change directions during connection period.

11) During low traffic period, the cell zooming server acts and activates sleep mode to save energy. During the entire day, the server uses the virtual cell zooming algorithm to improve system performance.

12) When cells are switched on or off, transition cost (e.g. handover) is not considered.

\section{B. Problem Formulation}

Simulations run on a 24 hour simulation time basis. The execution of sleep mode and virtual cell zooming algorithms must be compared with a standard, power-management-free operation of the simulated environment.

Sleep mode acts during low traffic period (0-9 a.m.), when cells are switched off in $(3,4)$-o ff scheme. To minimize the rise of the outage probability, active cells double their transmit power $(+3 \mathrm{~dB})$ and/or double their radius $(2 \times)$ by pointing their antennas to a more distant border.

Finally, we expect that with virtual cell zooming resources are better distributed, which can be shown on the analys is of users' throughput. Since virtual cell zooming operates during

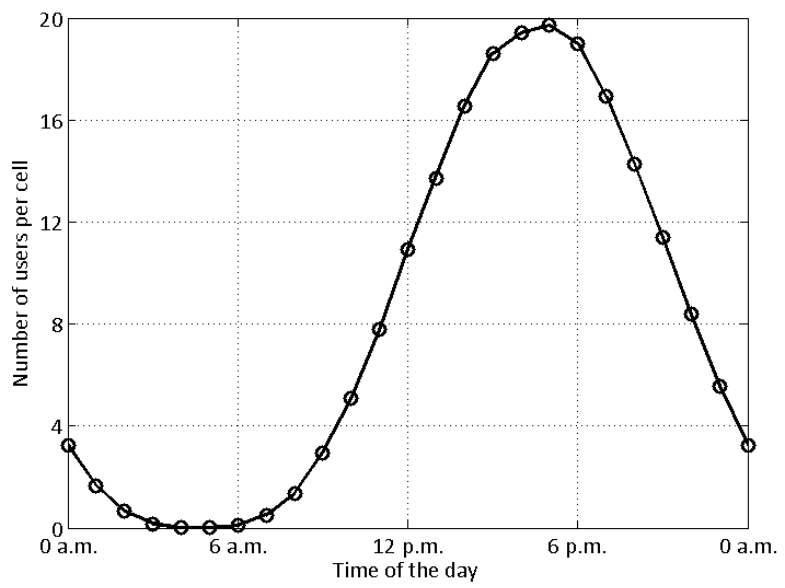

Fig. 3. Traffic profile during a day.

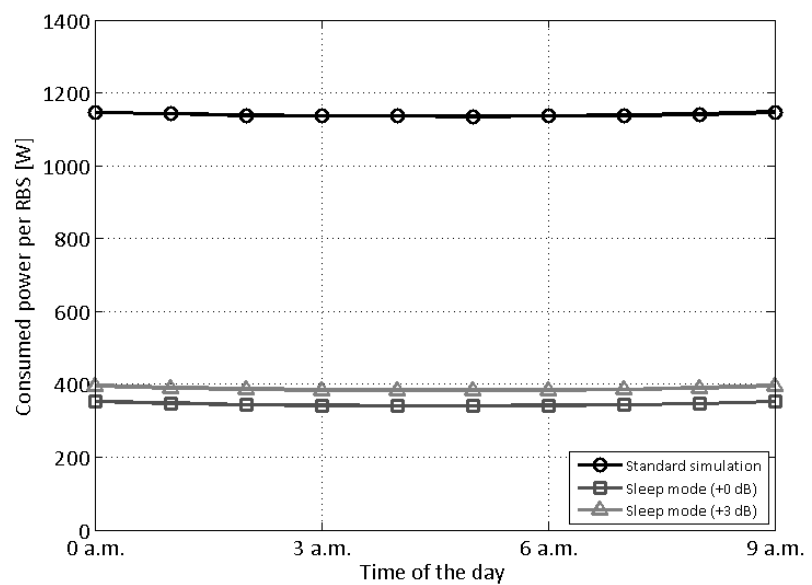

Fig. 4. Consumed power per base station from 0 to 9 a.m. in the standard simulation and in the sleep mode. the entire day, the throughput shall significantly grow, although consumed power will maintain the same.

Both algorithms are tested in different simulations in order to provide a separate analys is to each one.

\section{RESUlTS}

As seen before, the number of users existing in the grid varies sinusoidly, and simulation results as in Fig. 3. We set peak time to 5 p.m.

At first, we show how drastically power consumption is reduced when sleep mode is activated. During low traffic load, average consumed power per base station drops significantly to nearly $30 \%$ of the estimate in the standard simulation (Fig. 4). We must, however, investigate also what happens with system performance.

Since fewer cells are active during sleep mode, resources are distributed to many more users per cell. Hence, users' throughput also drops, but it still reaches acceptable levels (Fig. 5). Also, with fewer cells, uncovered areas may appear, and users may not establish connection with any of the RBSs, then raising outage probability. Nevertheless, Fig. 6 shows that by only adjusting antenna downtilt, outage is almost suppressed. As it is not necessary to adjust power level, energy efficiency is even better optimized.

Consumed power and outage probability (Figs. 7 and 8) remain unaltered for both standard and virtual cell zooming simulation. Although virtual cell zooming does not contribute

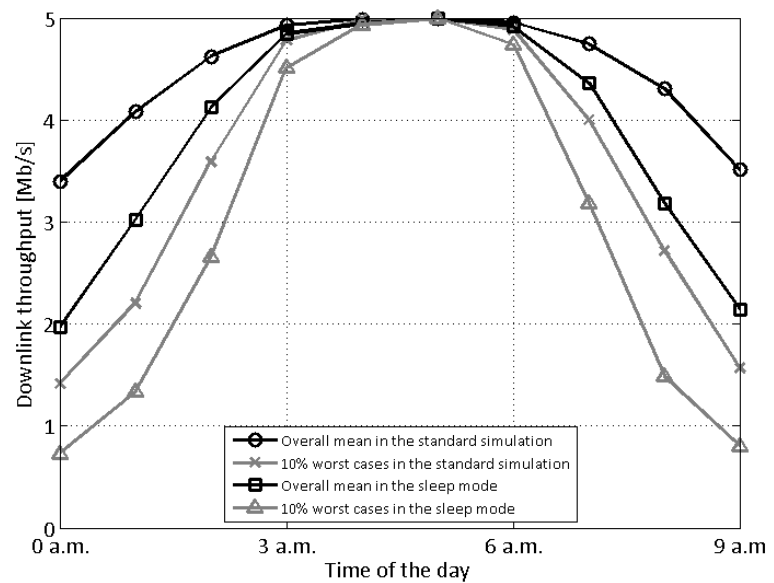

Fig. 5. Users' downlink throughput from 0 to 9 a.m. in the standard simulation and in the sleep mode.

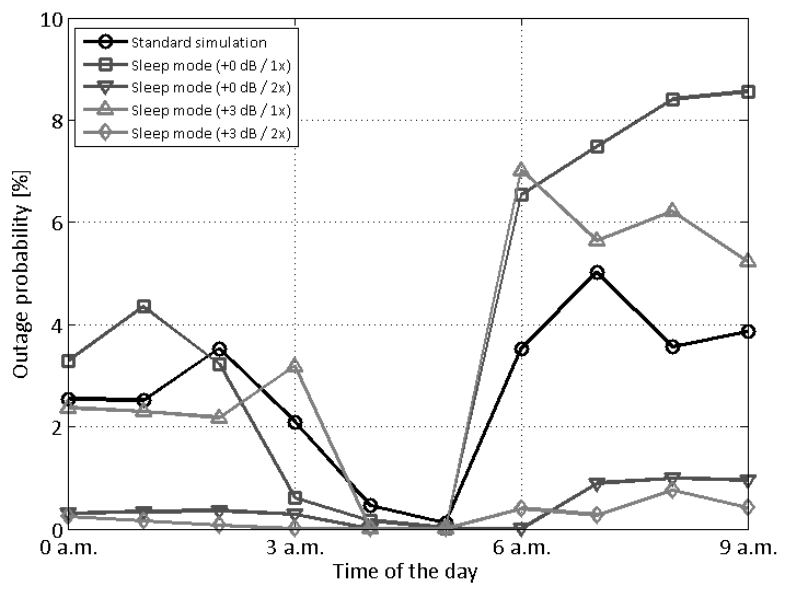

Fig. 6. Outage probability from 0 to 9 a.m. in the standard simulation and in the sleep mode. 


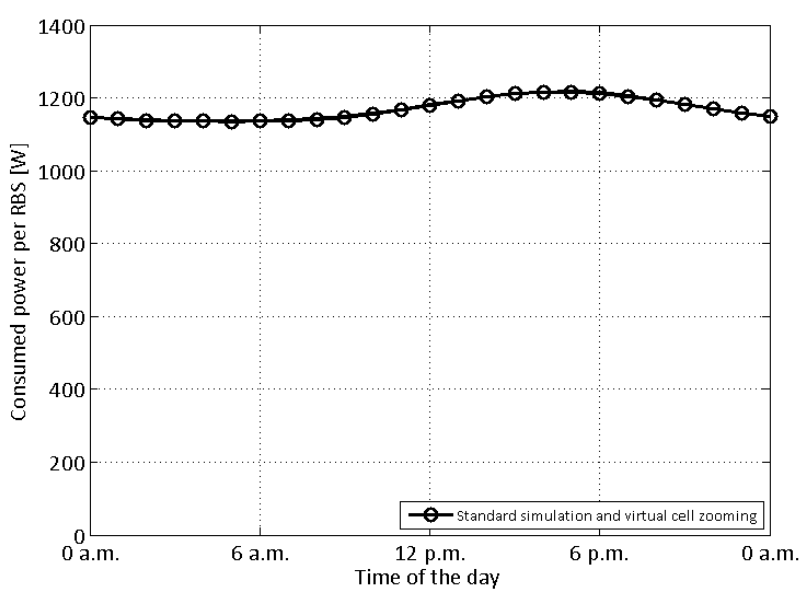

Fig. 7. Consumed power per base station during a day in the standard simulation and in the virtual cell zooming.

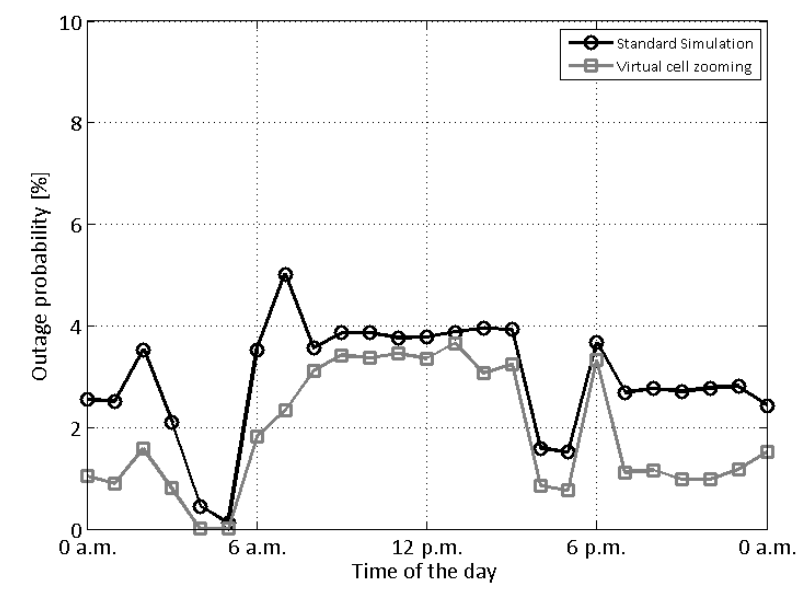

Fig. 8. Outage probability during a day in the standard simulation and in the virtual cell zooming.

to energy savings or coverage enhancements, throughput is highly improved with it (Fig. 9). Resources are better distributed because users are better distributed through cells. If a cell experience light traffic load while its neighbors are heavily loaded, resources from the former are being wasted. This waste is reduced by the virtual cell zooming technique.

\section{CONCLUSION}

A new vision of cell zooming is proposed in this article. The technique is separated into two, and we show that one - sleep mode - is better used for energy efficiency of cellular networks, and the other - virtual cell zooming - proves to be useful to enhance system performance by smoothly distributing network resources amongst mobile users.

The analysis does not consider extra processing to execute both algorithms, but as they run on a large time scale, in the order of minutes, it is not likely that their complexity will pose any implementation difficulties.

Moreover, in the near future we will probably rely on the use of smaller cells, and cons equently the deployment of numerous base stations. This is an ideal scenario to implement cell zoo ming algorithms in order to minimize energy consumption, and we have seen that the combination of sleep mode and virtual cell zooming can be a powerful method to achieve this goal.

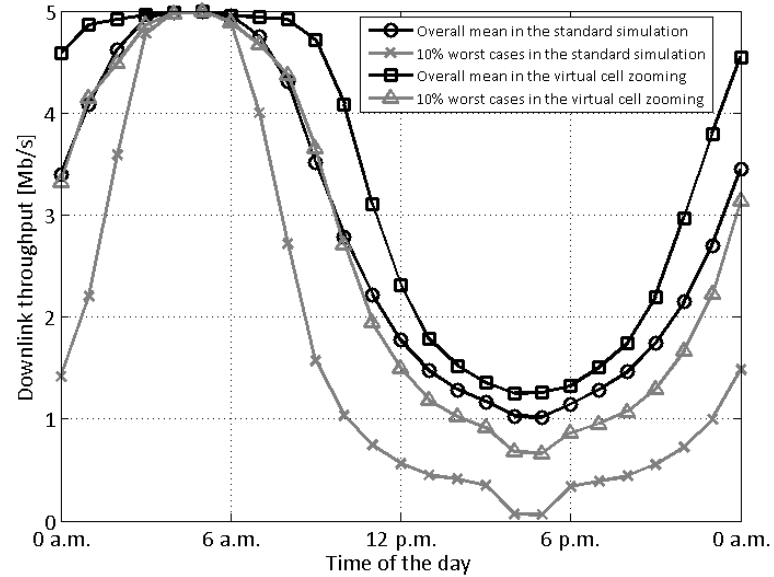

Fig. 9. Users' downlink throughput during a day in the standard simulation and in the virtual cell zooming.

\section{REFERENCES}

[1] E. Oh, B. Krishnamachari, X. Liu, and Z. Niu, "Toward Dynamic Energy-Efficient Operation of Cellular Network Infrastructure", IEEE Communications Magazine, pp. 56-61, Jun. 2011.

[2] Z. Hasan, H. Boostanimehr, and V. K. Bhargava, "Green Cellular Networks: A Survey, Some Research Issues and Challenges", IEEE Communications Surveys \& Tutorials, vol. 13, no. 4, pp. 524-540, 2011.

[3] Z. Niu, Y. Wu, J. Gong, and Z. Yang, "Cell Zooming for Cost-Efficient Green Cellular Networks”, IEEE Communications Magazine, pp. 74-79, Nov. 2010.

[4] X. Weng, D. Cao, and Z. Niu, "Energy-Efficient Cellular Network Planning under Insufficient Cell Zooming", 2011.

[5] T. S. Rappaport, Wireless Communications: Principles and Practice, 2nd ed., Prentice Hall, 2001.

[6] J. Zhuang, L. Jalloul, R. Novak, and J. Park, "Evaluation Methodology for P802.16m - Advanced Air Interface", IEEE 802.16 Broadband Wireless Access Working Group, pp. 44-45, Jan. 2009.

[7] F. Gunnarsson, M. N. Johansson, A. Furuskär, M. Lundevall, A. Simonsson, C. Tidestav, and M. Blomgren, "Downtilted Base Station Antennas - A Simulation Model Proposal and Impact on HSP A and LTE Performance", 2008. 